\title{
LE COEUR-ESPACE: ASPECTS DU LYRISME \\ DANS LES FLEURS DU MAL
}

\section{Michel Collot}

Si l'on a célébré un peu partout en 2007 le cent-cinquantième anniversaire des Fleurs du Mal, c'est qu'on reconnait en elles un sommet de la poésie française, et un moment important de son histoire. Toute une part de la réception moderne et contemporaine est pourtant assez critique à leur égard, et tend à leur préférer par exemple Le Spleen de Paris, ce titre que Michel Deguy n'a pas hésité à emprunter à Baudelaire. Beaucoup, comme déjà Rimbaud, trouvent leur forme" mesquine"1." Et Jean-Marie Gleize dit préférer pour les "chiens" de la prose baudelairienne aux chats qui hantent les vers des Fleurs du Mal.

Or n'est-ce pas précisément grâce à leur forme: forme versifiée, forme fixe, souvent brève, que les poèmes qui les composent s'impriment durablement dans la mémoire? Et d'ailleurs, cette forme n'est-elle pas aujourd'hui redevenue d'actualité? Beaucoup de poètes français ont redécouvert ces dernières années les vertus du vers, qu'il s’agisse de réhabiliter le vers régulier, comme le fait Jacques Réda, ${ }^{2}$ ou d'inventer de nouvelles formes de versification, comme s'y essaient, entre autres, Pierre Alféri ou Philippe Beck.

Mais la forme ne suffit pas à expliquer la fortune des Fleurs $d u$ Mal. Si certains d'entre nous les connaissent encore par cœur, c'est parce qu'elles parlent à notre cœur, se logent au cœur de notre conscience la plus intime. Et si elles ont franchi les frontières et survécu à l'épreuve de la traduction, c'est que leur résonance n'est pas seulement liée à leur consonance, au rythme, à l'harmonie et à la mélodie de leurs vers, mais aussi à leur sens, capable de toucher le cour humain dans ce qu'il a de plus universel.

Voilà des qualités éminemment classiques, et c'est une autre cause de la longévité des Fleurs $d u$ Mal, qui ont résisté aux modes éphémères; mais c'est aussi peut-être une des raisons de leur actualité: beaucoup d'esprits déçus par le modernisme retrouvent aujourd'hui les voies d'un certain classicisme, qui n'est pas

\footnotetext{
1 "La forme si vantée en lui est mesquine".

${ }^{2}$ Voir le titre d'un de ses récents recueils: L'Adoption du système métrique (Paris: Gallimard, 2004).
}

"(DEGUY, Michel. Spleen de Paris, Galilée, 2001.)

(RIMBAUD, Arthur. Lettre à Paul Demeny du 15 mai 1871. Dans CEuvres complètes d'Arthur Rimbaud. Bibliothèque Numérique de I'Université Paris VIII.) 
- (BAUDELAIRE, Charles. Salon de 1846. Dans CEuvres complètes. Bibliothèque de la Pléiade. Paris: Gallimard, 1976: II, 421.) incompatible avec la plus authentique modernité: celle qui, selon l'enseignement de Baudelaire, vise l'éternel à travers le transitoire.

Baudelaire n'a pas seulement été le premier à donner au terme de modernité ses lettres de noblesse, et sa définition la plus profonde, il a, par sa pratique elle-même, ouvert de multiples voies à la poésie moderne, comme en témoigne suffisamment l'influence qu'il a eue depuis un siècle et demi, sur des poètes aussi importants et aussi différents que Rimbaud et Mallarmé, ou Bonnefoy et Deguy, par exemple, pour me limiter à la France.

Cette modernité de Baudelaire a été diversement interprétée. Beaucoup de commentateurs la trouvent plutôt dans sa prose, poétique ou critique. Il n'est pas toujours facile de la repérer dans Les Fleurs du Mal, sans doute parce qu'elle s'y trouve étroitement mêlée à l'héritage romantique. Je rappelle que, pour Baudelaire, "Qui dit romantisme dit art moderne, - c'est-à-dire intimité, spiritualité, couleur, aspiration vers l'infini, exprimées par tous les moyens que contiennent les arts". ' Or le modernisme a eu tendance à rejeter la plupart de ces valeurs romantiques, jugées suspectes de lyrisme naïf ou d'idéalisme. Du coup, c'est tout un versant de la poétique des Fleurs du Mal, celui de l'Idéal, qui se trouverait frappé de caducité, et que je me propose de revisiter et de réévaluer. Car, à mes yeux, comme aux yeux d'un certain nombre de mes contemporains, la modernité la plus profonde trouve sa source dans le romantisme. Il y a même aujourd'hui une certaine actualité du romantisme, à condition de ne pas le confondre avec la caricature qu'en ont parfois donnée les avant-gardes du siècle dernier. ${ }^{3}$

\section{Le lyrisme hors de soi}

Parmi les traits que Baudelaire hérite du romantisme, figure un certain lyrisme, qu'il ne faut pas se hâter de confondre avec l'expression du sentiment personnel, ou d'une pure intériorité, comme le faisait Hegel, en l'opposant à l'objectivité de la poésie épique, tournée vers l'extérieur. Le lyrisme romantique n'est pas toujours l'effet d'une sorte d'inflation du moi, qui projetterait dans les mots et dans les choses ses états d'âme. Un poète réputé égocentrique comme Byron écrit par exemple: "I live not in myself, but I becomel Portion

\footnotetext{
${ }^{3}$ J'ai fait le point sur cette "Actualité du romantisme", dans un article paru dans Le Nouveau Recueil, n 77 , déc.2005 - fév. 2006: 153-159.
} 
of that around me; and to me/High mountains are a feeling". ${ }^{*}$ C'est un tel transport du dedans vers le dehors qui définit, selon Baudelaire, la "manière lyrique de sentir": "tout l'être intérieur" "s'élance en l'air par trop de légèreté et de dilatation, comme pour atteindre une région plus haute", écrit-il. " Il me semble qu'on assiste souvent dans Les Fleurs du Mal à un tel espacement du sujet, qui ouvre une voie nouvelle et féconde à la poésie moderne. ${ }^{5}$

On résume trop souvent l'évolution de celle-ci à une alternative entre un lyrisme censé faire la part belle à la subjectivité, et un "littéralisme" qui tendrait vers l'objectivité. Ce clivage sommaire laisse de côté une troisième voie: celle d'un lyrisme moderne, qui n'est plus l'expression d'une identité et d'une intériorité, mais celle d'une altérité et d'une extériorité. C'est celle qu'emprunte Rimbaud quand il annonce que "JE est un autre"; et Mallarmé lui-même, s'il proclame la "disparition élocutoire du poète" n'exclut pas d'en exprimer l'affectivité par le biais des mots et des choses. "Évoquer petit à petit un objet pour montrer un état d'âme, ou inversement choisir un objet et en dégager un état d'âme", telle sera la démarche fondamentale de la poésie symboliste, et c'est déjà la définition que Baudelaire donne de l'art moderne: "Qu'est-ce que l'art pur suivant la conception moderne? C'est créer une magie suggestive contenant à la fois l'objet et le sujet, le monde extérieur à l'artiste et l'artiste lui-même". Au sujet lyrique correspond donc, selon Baudelaire, "un monde lyrique": "une atmosphère lyrique, des paysages, des hommes, des femmes, des animaux qui tous participent du caractère affectionné de la lyre". *

Hugo Friedrich n'a pas tort de parler, à propos de Baudelaire, d'une "dépersonnalisation"; " mais celle-ci n’aboutit pas à la disparition du lyrisme: plutôt à sa transformation. Dans Les Fleurs du Mal, le sujet lyrique s'arrache parfois aux limites de son identité personnelle pour s'identifier à un objet ou à un lieu, selon un mouvement qui caractérise, selon Baudelaire, l'ivresse poétique ou celle procurée par le haschich: "Il arrive parfois que la personnalité disparâ̂t et que l'objectivité (...) se développe en vous si anormalement que la contemplation des objets extérieurs vous fait oublier votre propre existence et que vous vous confondez bientôt avec eux (...)".* Particulièrement frappante est, à cet égard, l'association récurrente

\footnotetext{
4 "Je ne vis pas en moi-même, mais je deviens/ Une partie de ce qui m'entoure; et pour moi/ Les hautes montagnes sont une émotion".

${ }^{5}$ Voir mon essai Paysage et Poésie, Corti, 2005: 43-64.
}

* (BYRON, Lord Georg Gordon. Childe Harold's Pilgrimage, Chant III, strophe 72, traduction Roger Martin. Paris: Aubier-Montaigne, 1949: 207.)

* (BAudelaire, C. Réflexions sur quelques-uns de mes contemporains. Dans OEuvres complètes. Op. cit.: II, 164.)

* (MALLARMÉ, Stéphane. Sur l'Évolution littéraire. Dans CEuvres complètes. Bibliothèque de la Pléiade. Paris: Gallimard, 2003: II, 700.)

* (BAUDELAIRE, C. L'Art philosophique. Dans $C E$ vres complètes. Op. cit.: II, 598.)

* (BAudelaire, C. Réflexions sur quelques-uns de mes contemporains. Op. cit.: II, 598.)

(FRIEDRICH, Hugo. Structure de la poésie moderne. Trad. Michel-François Demet. Paris: Le Livre de Poche, 1999.)

(BAudelaire, C. Le Poème du haschisch. Dans OEuvres complètes. Op. cit.: I, 419.) 
du syntagme "Je suis" avec un nom d'objet placé en position d'attribut du sujet, comme au début du poème consacré à La Pipe: "Je suis la pipe d'un auteur". ${ }^{*}$ Pareille construction est assez rare dans la poésie antérieure; et surtout elle reste en général ponctuelle, alors que l'identification du Je à la pipe se poursuit tout au long du poème. On m’objectera qu'ici l'on a affaire au procédé classique de la prosopopée, qui consiste à faire parler un objet inanimé. Ce n'est pas le cas dans d'autres poèmes des Fleurs $d u$ Mal, où le Je semble bien représenter le poète, qui s'identifie successivement à plusieurs lieux ou objets. Ainsi dans l'un des Spleen, où chaque identification se développe en description:

Je suis un cimetière abhorré de la lune,

Où, comme des remords se traînent de longs vers

Qui s'acharnent toujours sur mes morts les plus chers. Je suis un vieux boudoir plein de choses fanées,

Où gît tout un fouillis de modes surannées,

Où les pastels plaintifs et les pâles Boucher,

Seuls, respirent l'odeur d'un flacon débouché.

Dans L'Héautontimoroumenos, les identifications se multiplient et se succèdent sur un rythme accéléré:

Je suis le sinistre miroir

Où la mégère se regarde.

Je suis la plaie et le couteau!

Je suis le soufflet et la joue!

Je suis les membres et la roue!

De par sa répétition vertigineuse, une tournure qui sert habituellement à décliner l'identité en exprime ici éloquemment la crise; non seulement le sujet se dédouble, à la fois victime et bourreau de lui-même, mais il se pluralise et se déshumanise, en s'assimilant aussi à toute une série d'instruments de torture. On remarquera que cette tournure n'est pas réservée à la première personne; elle s'étend, en troisième personne, aux métonymies du poète, comme le cœur, et, en seconde personne, à ses destinatrices: on lit par exemple, dans Causerie, "Mon cœur est un palais flétri par la cohue" et "Vous êtes un beau ciel d'automne, clair et rose".

\footnotetext{
${ }^{6}$ Les références des citations extraites des Fleurs $d u$ mal seront indiquées entre parenthèses par la mention FDM suivie de la numérotation du poème dans l'édition de 1861, et de la pagination de l'édition Gallimard-Pléiade.
} 
On pourrait certes considérer cette identification d'un sujet humain à un lieu ou à un objet comme un simple procédé rhétorique, relevant de la métaphore ou de l'allégorie, destiné à concrétiser une idée abstraite ou un sentiment. Il me semble que l'insistance de cette tournure, et le développement qu'elle prend dans certains poèmes des Fleurs $d u$ Mal, lui donne une portée plus essentielle, qui remet en cause la distinction entre le concret et l'abstrait.

Le fonctionnement traditionnel de l'allégorie repose sur une correspondance terme à terme entre comparé et comparant, qui subordonne clairement celui-ci à celui-là. C'est le cas par exemple de l'analogie entre visage et paysage développée dans $\grave{A}$ celle qui est trop gaie:

Ta tête, ton geste, ton air

Sont beaux comme un beau paysage;

Le rire joue en ton visage

Comme un vent frais dans un ciel clair.

À ce fonctionnement logique et analytique se substitue dans certains cas un fonctionnement affectif et synthétique, qui finit par rendre indistincts comparé et comparant, comme dans le vers de Causerie cité plus haut. Le passage d'un régime à l'autre est exemplairement perceptible dans Ciel brouillé; au début, la distinction est nette entre le visage féminin et le ciel auquel il est comparé, mais elle s'estompe progressivement, au point qu'on ne sait plus si le poète s'adresse à la femme ou au paysage, qui devient allocutaire et passe au premier plan, comme l'annonçait le titre du poème:

Tu ressembles parfois à ces beaux horizons

Qu'allument les soleils des brumeuses saisons...

Comme tu resplendis, paysage mouillé

Qu'enflamment les rayons tombant d'un ciel brouillé!

On assiste ici à l'expansion et à l'autonomisation du terme comparant, qui finit par occuper tout l'espace de la strophe: l'image de départ se développe en un véritable tableau. Ce phénomène récurrent dans Les Fleurs du Mal ne révèle pas seulement l'habileté rhétorique de Baudelaire, ni son goût pour la peinture, ni même la puissance de son imagination. Il correspond à une expérience, souvent évoquée et invoquée par Baudelaire comme le fondement même de l'allégorie, et qui se caractérise par une totale identification du sujet à l'objet: 
(BAUDELAIRE, C. Le Poème du haschisch. Op. cit.: 419-420.)

- (BAUDELAIRE, C. Fusées, XI. Dans CEuvres complètes. Op. cit.: I, 659.)

(DEGUY, M. Actes. Paris: Gallimard, 1966: 256.)

(BAUDELAIRE, C. Le Poème du haschisch. Op. cit.: $\mathrm{I}, 430$.)

$\cdot($ Idem)

(BAUDELAIRE, C. Le Spleen de Paris, III. Dans CEuvres complètes. Op. cit.: I, 278.)
Votre œil se fixe sur un arbre courbé par le vent; dans quelques secondes, ce qui ne serait dans le cerveau d'un poète qu'une comparaison fort naturelle deviendra dans le vôtre une réalité. Vous prêtez d'abord à l'arbre vos passions, votre désir ou votre mélancolie; ses gémissements et ses oscillations deviennent les vôtres, et bientôt vous êtes l'arbre. De même, l'oiseau qui plane au fond de l'azur représente d'abord l'immortelle envie de planer au-dessus des choses humaines; mais déjà vous êtes l'oiseau lui-même.

Cet échange entre le dedans et le dehors qu'exprime la métaphore, le symbole ou l'allégorie se réalise dans certains états favorables à l'intériorisation du monde extérieur ou à l'extériorisation de la vie intérieure: "Dans certains états de l'âme, presque surnaturels, la profondeur de la vie se révèle tout entière dans le spectacle, si ordinaire qu'il soit, qu'on a sous les yeux. Il en devient le symbole". " Commentant cette note de Fusées, Michel Deguy souligne que, malgré leur caractère exceptionnel, qualifié même de "surnaturel", de telles expériences reposent sur un phénomène "naturel", que l'on "a sous les yeux": il ne s'agit pas de "sortir de ce monde", mais "de l'immanence se révélant dans sa propre dimension énigmatique”. "C'est donc du sensible lui-même qu'émerge un sens, qui fait du "premier objet venu" un "symbole parlant".

Ainsi comprise comme sensibilisation du sens ou sémantisation du sensible, l'allégorie apparaît comme "l'une des formes primitives et les plus naturelles de la poésie", et elle n'a pas besoin pour se développer de l'action d'un psychotrope. Elle peut naître par exemple de la contemplation d'un paysage, comme au début du Confiteor de l'artiste:

Grand délice que celui de noyer son regard dans l'immensité du ciel et de la mer! Solitude, silence, incomparable chasteté de l'azur! une petite voile frissonnant à l'horizon, et qui par sa petitesse et son isolement imite mon irrémédiable existence, mélodie monotone de la houle, toutes ces choses pensent par moi, ou je pense par elles (car dans la grandeur de la rêverie, le moi se perd vite!); elles pensent, dis-je, mais musicalement et pittoresquement, sans arguties, sans syllogismes, sans déductions.

\section{L'espacement du sujet}

Ce texte illustre le mouvement proprement ek-statique qui fait sortir le sujet lyrique des limites du moi, ce transport qui est au fondement du transfert de sens à l'œuvre dans la métaphore. Le chiasme: "toutes ces choses pensent par moi, ou je pense par elle" 
souligne cette "intrication du monde intérieur et du monde extérieur" qui caractérise, selon Emil Staiger, le lyrisme, et qui trouve dans le paysage une expression privilégiée. Dans un célèbre passage du Salon de 1859, Baudelaire a insisté, contre le réalisme qui prétend peindre "l'univers sans l'homme", sur la part qui revient au sujet dans l'art du paysage:

Si tel assemblage d'arbres, de montagnes, d'eaux et de maisons, que nous appelons un paysage, est beau, ce n'est pas par lui-même, mais par moi, par ma grâce propre, par l'idée ou le sentiment que j'y attache.

Il ne s'agit pas pour autant d'opposer à la prétendue objectivité des réalistes un pur subjectivisme: c'est de "la masse suggestive éparpillée dans l'espace”, de "(s)a qualité inspiratrice” que le paysagiste "extrait" "la comparaison, la métaphore et l'allégorie", et tout son art consiste à "traduire un sentiment par un assemblage de matière végétale ou minérale". "La "matière-émotion" du poème ou du tableau résulte d'une fusion entre la matière du monde et les "impressions" qu'elle éveille, car "de prodigieuses rêveries sont contenues dans les spectacles de la nature".

Le paysage nait d'un double mouvement de projection du sujet dans le monde et d'introjection des objets par la conscience. C'est un espace subjectif habité par un sujet qui s'espace. "Lesprit s'enfonce" "dans le ciel, dans l'horizon de la mer" comme "dans des yeux pleins de pensée" ou "dans une tendance féconde et grosse de rêverie", écrit Baudelaire à propos de Delacroix. Dans l'expérience et dans l'art du paysage, une pensée est à l'œuvre, qui ignore la distinction cartésienne entre la chose pensante et la chose étendue: une pensée dans l'espace, une pensée-paysage.

On peut la rapprocher de cette "pensée fondamentale" que Merleau-Ponty décèle dans l'expérience sensible, et qui ne peut être traduite par les "arguties", les "syllogismes" et les "déductions du discours, mais par un langage rendu lui-même sensible, comme celui de la peinture ou de la poésie, "musicalement" ou "pittoresquement". "Toutes ces choses pensent par moi, ou je pense par elles”: telle pourrait être la formule de ce cogito pré-réflexif qui est aussi à l'œuvre dans l'espacement du sujet lyrique.

On m'objectera que, chez Baudelaire, ce mouvement ou ce moment lyrique est toujours menacé par une réflexion critique qui rétablit la distinction et parfois l'opposition entre le moi et le monde. Ainsi, dans Le Confiteor de l'artiste, à l'extase lyrique suc- 
• (BAUDELAIRE, C. "À Arsène Houssaye", Le Spleen de Paris. Op. cit.: I, 275-276.)

(FDM III: 10)

(FDM LXXVII: 74)

(FDM éd. de 1868: 142)

(COLLOT, M. "Horizon et imagination". Dans Paysage et Poésie, du romantisme à nos jours. Paris: José Corti, 2005: 65.)

(BAUDELAIRE, C. Mon cœur mis à nu. Dans CEuvres complètes. Op. cit.: I, 677.) cède l'“exaspération" du poète face à "l'insensibilité de la mer" et à "l'immuabilité d'(un) spectacle" dont il ne parvient pas à exprimer la beauté et l'intensité.

L'identification du sujet à l'univers ne serait-elle qu'une illusion lyrique? La composition de ce texte oppose à "la grandeur de la rêverie" la conscience lucide des limites de l'humaine condition. La force et la modernité des poèmes en prose de Baudelaire résident dans cette confrontation constante entre lyrisme et réalisme. La prose permet d'exprimer à la fois "les mouvements lyriques de l'âme", "les ondulations de la rêverie" et "les soubresauts de la conscience", qui rétablit les distinctions logiques et rappelle la résistance d'une "réalité rugueuse à étreindre".

Il me semble que le lyrisme s'épanouit plus librement dans Les Fleurs du Mal. En témoigne par exemple l'insistance du mot cœur, qui y revient 117 fois, contre seulement 13 occurrences dans Le Spleen de Paris. ${ }^{7}$ Non que la conscience réflexive y soit absente; mais le souci de l'harmonie du vers, de l'unité du poème et de la construction du recueil a conduit Baudelaire à éviter le mélange des tons, et à répartir par exemple Spleen et Idéal en deux séries distinctes. De ce fait, certains poèmes des Fleurs du Mal, comme Le Balcon, Élévation, La vie antérieure, Harmonie du soir sont de purs moments lyriques, où se donne libre cours d'un bout à l'autre l'espacement du sujet. Celui-ci n'est d'ailleurs bien sûr pas réservéà l'expression d'une fusion bienheureuse avec l'autre et avec le monde; il peut être aussi bien douloureux: au "libre essor" dans "l'air supérieur" de l'Idéal " s'oppose "le ciel bas et lourd" du Spleen, qui "pèse comme un couvercle/ Sur l'esprit gémissant". Euphorique ou dysphorique, l'état d'âme se transforme en paysage; et réciproquement, l'espace s'intériorise au point de devenir inséparable du sujet: "Pascal avait son gouffre, avec lui se mouvant".

La plupart des commentateurs qui ont pris au sérieux cette spatialisation des affects ont mis l'accent sur la verticalité de l'espace baudelairien, polarisé par l'appel du ciel et l'attirance de l'abîme. Mais la profondeur baudelairienne est aussi horizontale, comme je l'ai montré ailleurs, "et à la dialectique de l'ascension et de la chute, s'ajoute une autre, celle de l'expansion et de la restriction, qui est tout aussi importante, comme en témoigne cette note célèbre: "De la vaporisation et de la centralisation du Moi. Tout est là". Baudelaire reprend sans doute ici l'opposition qu'il avait rencon-

\footnotetext{
${ }^{7}$ Statistique établie d'après la base de données Frantext de l'ATILF (CNRS).
} 
trée chez Emerson entre "dissipation" et "concentration". Pour"la conduite de la vie" (c'est le titre de l'essai du philosophe américain auquel Baudelaire se réfere), l'homme doit se garder des tentations qui le détournent de lui-même. Baudelaire, on le sait, critique ce "besoin de sortir de soi" qui apparente l'amour lui-même à une "prostitution"; l'artiste, à ses yeux, ne doit "jamais" "sort(ir) de lui-même".

Pourtant, bien des poèmes des Fleurs du Mal nous font assister à une telle sortie de soi, et la "vaporisation" n'y est pas toujours aussi négative qu'elle ne l'est dans l'adresse liminaire "Au lecteur", où elle apparaît comme l'œuvre de Satan. Elle prend souvent la forme d'une expansion positive, alors que la concentration aboutit parfois à une introspection douloureuse ou à un tête-à-tête destructeur avec soi-même, quand elle ne s'accompagne pas d'une constriction oppressante de l'espace et du corps. Tout se passe comme si le lyrisme supposait cette sortie et cette vaporisation de soi dont le Moi ne veut pas, et comme si Baudelaire valorisait, sur le plan poétique et esthétique, ce qu'il dénonce sur le plan moral et intellectuel. Je me propose à présent d'étudier plus précisément quelques-unes des modalités et expressions exemplaires de cet espacement du sujet dans les Fleurs du Mal.

\section{Le cour-espace}

Je reviens d'abord sur la place qu'occupe le cœur dans le recueil. L'insistance de ce mot et de ce motif pourrait manifester la fidélité de Baudelaire à un lyrisme assez traditionnel, qui n'est d'ailleurs pas absent de certains poèmes des Fleurs du Mal. Le cour est en effet l'organe par excellence de l'intimité et de l'intériorité. Dans plusieurs poèmes, il se fait au contraire l'instrument de l'espacement et du décentrement du sujet lyrique; il devient par exemple dans Causerie un lieu public, "un palais flétri par la cohue". Alors qu'il devrait occuper le centre de la vie affective, il se trouve parfois projeté dans l'espace: le "cœur" du voyageur en route pour Cythère, "comme un oiseau/ Voltig(e) tout joyeux/ Et plan(e) librement à l'entour des cordages";" et celui d'Agathe "s'envole" loin de la "cité" qu'elle habite "Vers un autre océan où la splendeur éclate".

Il devient plus souvent encore un lieu d'échange entre le dedans et le dehors. Ainsi, dans Chant d'automne, le "cour" du poète, envahi par l'atmosphère hivernale qui l'entoure, se retrouve lui-même transformé en paysage polaire: 
Tout l'hiver va rentrer dans mon être: colère,

Haine, frissons, horreur, labeur dur et forcé,

Et, comme le soleil dans son enfer polaire,

Mon cœur ne sera plus qu'un bloc rouge et glacé.

(BONNEFOY, Yves. Le coeur-espace (1945-1961). Paris: Farrago/ Léo Scheer, 2001.)

(FDM XXXVI: 37.$)$
•(CHRÉTIEN, Jean-Louis. La Joie spacieuse. Paris: Minuit, 2007: 8.)
•(FDM LLVII: 47.)
Il n'appartient plus dès lors au seul poète, comme en témoigne souvent la disparition de l'adjectif possessif au profit d'un simple article, défini ou indéfini;"le "cœur apparaît ainsi dans Le Balcon comme le foyer partagé d'un espace indistinctement intérieur et extérieur, véritable "cœur-espace", pour reprendre le beau titre d'un des tout premiers essais poétiques d'Yves Bonnefoy:

Que les soleils sont beaux dans les chaudes soirées! Que l'espace est profond, que le cœur est puissant!

Commentant ces deux vers, le philosophe Jean-Louis Chrétien les commente en ces termes: "l'épreuve de la joie est toujours une épreuve de l'espace en crue. Espace du soi, espace du monde? Espace intérieur, espace extérieur? Le propre de la joie est de rendre cette distinction caduque, et d'être indivisément une épreuve de soi et une épreuve du monde. Nul ne l'a mieux dit que Baudelaire dans ces vers du Balcon. (...) C'est seulement quand l'espace s'approfondit que le cœur se renforce, et c'est seulement quand le cœur se renforce que l'approfondissement de l'espace nous est donné à voir et à vivre". *

Mais cet effacement des frontières entre l'intérieur et l'extérieur n'est pas réservé à la joie seule; il traduit la "puissance" de tout affect capable par son intensité de colorer le monde; ainsi de la mélancolie qui s'empare d'"un cœur tendre", et qui, dans Harmonie du soir, s'étend progressivement, au gré des répétitions obsédantes des mêmes mots et des mêmes images, au paysage tout entier:

Le violon frémit comme un cœur qu'on afflige;

Un cœur tendre, qui hait le néant vaste et noir!

Le ciel est triste et beau comme un grand reposoir;

Le soleil s'est noyé dans son sang qui se fige.

Un cœur tendre, qui hait le néant vaste et noir,

Du passé lumineux recueille tout vestige!

Le soleil s'est noyé dans son sang qui se fige...

Ton souvenir en moi luit comme un ostensoir!* 
L'apparition du pronom moi au dernier vers semble rapporter cette coloration affective à un drame personnel; mais on notera que le poète se garde de s'attribuer ce cœur, qui est précédé d'un article indéfini, et qui tend à se confondre avec le soleil, "noyé dans son sang". Cette hémorragie est la traduction physique de la tendance du sujet lyrique à se répandre au-dehors; elle est mise en scène de façon spectaculaire dans La Fontaine de sang:

Il me semble parfois que mon sang coule à flots, Ainsi qu'une fontaine aux rythmiques sanglots.

Je l'entends bien qui coule avec un long murmure, Mais je me tâte en vain pour trouver la blessure.

À travers la cité, comme dans un champ clos, Il s'en va, transformant les pavés en îlots, Désaltérant la soif de chaque créature, Et partout colorant en rouge la nature.

Les tercets font état de la "terreur" qu'inspire au poète cet épanchement involontaire, que les quatrains évoquent pourtant dans des termes étonnamment positifs. Plutôt qu'une "blessure", inexistante, ce "sang" qui "coule à flots" semble manifester une surabondance de vie, capable de régénérer la "nature" environnante. S'il fait entendre des "sanglots", ces derniers sont "rythmiques" et se résorbent en "un long murmure" analogue à celui du poème luimême. Le lyrisme n'est-il pas lui-même une effusion?

Une autre modalité de l'effusion lyrique est la vaporisation, que Baudelaire dit redouter dans ses écrits intimes, mais qu'il semble cultiver dans Les Fleurs du Mal. Si, dans un poème emblématique, le Crâne reproche au dieu Amour d'“éparpiller en l'air" "(s)a cervelle,/ (S)on sang et (s)a chair" pour en faire des bulles de savon, cette vaporisation s'apparente à une sublimation et s'accompagne d'une ascension, certes éphémère, mais brillante; "(l)es bulles rondes" "montent dans l'air,/ Comme pour rejoindre les mondes/ Au fond de l'éther":

Le globe lumineux et frêle

Prend un grand essor,

Crève et crache son âme grêle

Comme un songe d'or. 
C'est un phénomène analogue qui se produit par l'entremise de la pipe, dont les volutes de fumée semblent exercer une influence bénéfique sur les pensées de l'auteur:

J'enlace et je berce son âme

Dans le réseau mobile et bleu

Qui monte de sa bouche en feu,

Et je roule un puissant dictame

Qui charme son cœur et guérit

·(FDM LXVIII: 67.)

(BAUDELAIRE, C. Le Poème du haschisch. Op. cit: I, 420.)

De ses fatigues son esprit.

Dans Le Poème du hachisch, Baudelaire assimile cette opération à une véritable distillation de la pensée qui cesse d'appartenir au sujet et se résorbe dans l'atmosphère:

Je vous suppose assis et fumant. Votre attention se reposera un peu trop longtemps sur les nuages bleuâtres qui s'exhalent de votre pipe. L'idée d'une évaporation, lente, successive, éternelle, s'emparera de votre esprit, et vous appliquerez bientôt cette idée à vos propres pensées, à votre matière pensante. Par une équivoque singulière, par une espèce de transposition ou de quiproquo intellectuel, vous vous sentirez vous évaporant, et vous attribuerez à votre pipe (dans laquelle vous vous sentez accroupi et ramassé comme le tabac) l'étrange faculté de vous fumer."

La création poétique elle-même est une sorte de vaporisation, si l'on en croit l'image finale de Paysage, où le poète, se retranchant de l'ambiance urbaine et hivernale qui l'entoure se propose

D'évoquer le Printemps avec (s)a volonté

De tirer un soleil de (s)on cour, et de faire

(FDM LXXXVI: 82.)

Atmosphère, atmosphère

Ce terme d'atmosphère, familier à Baudelaire, est sans doute l'un des plus appropriés pour désigner l'espace dont s'entoure le sujet lyrique, puisqu'il évoque à la fois une composante physique du paysage et sa coloration affective. C'est une aire transitionnelle, propice aux interactions entre le dedans et le dehors. Baudelaire, comme beaucoup de ses contemporains, se montre sensible à l'influence du climat sur l'humeur et les états d'âme. "Le ciel bas 
et lourd pèse comme un couvercle/ Sur l'esprit gémissant en proie aux longs ennuis"*', mais il peut aussi procurer à un cœur blessé une enveloppe protectrice:

Ô fins d'automne, hivers, printemps trempés de boue Endormeuses saisons! je vous aime et vous loue

D'envelopper ainsi mon cœur et mon cerveau

D'un linceul vaporeux et d'un vague tombeau. ${ }^{*}$

Réciproquement, la vie intérieure présente "à ceux qui savent l'observer eux-mêmes et qui gardent la mémoire de leurs impressions", des fluctuations semblables à celles de la météorologie, et Baudelaire loue Hoffmann d'avoir inventé "un singulier baromètre psychologique destiné à lui représenter les différentes températures et les phénomènes atmosphériques de son âme". *

Dans sa dimension la plus concrète, l'atmosphère résulte de l'interaction entre l'air, l'eau et la lumière, qui sont les éléments privilégiés du paysage baudelairien, sans doute parce qu'ils sont les moins chargés de matérialité et donc les plus aptes à exprimer des valeurs affectives ou spirituelles. Il excelle à évoquer aussi bien un "ciel brouillé"* que "le feu clair qui remplit les espaces limpides" "dans l'air supérieur". * Critique d'art, Baudelaire se montre particulièrement attentif au rendu de l'air et de la lumière, sans lesquels un paysage est privé de vie et de poésie: "La ligne et le style ne remplacent pas la lumière, l'ombre, les reflets et l'atmosphère colorante, toutes choses qui jouent un trop grand rôle dans la poésie de la nature pour qu'elle se soumette à (la) méthode" des disciples d'Ingres. Les mêmes qualités sont requises dans l'art du portrait, si l'on veut en faire "un poème" "plein d'espace et de rêverie", car l'expression d'un visage est inséparable de l'aura qui l'entoure: "Il faut savoir baigner une tête dans les molles vapeurs d'une chaude atmosphère, ou la faire sortir des profondeurs d'un crépuscule". *

L'atmosphère d'un intérieur est elle aussi apte à créer autour du sujet lyrique un climat affectif; elle est d'ailleurs souvent associée à celle qui baigne le paysage environnant. Aux "soirs au balcon, voilés de vapeurs roses" répondent "les soirs illuminés par l'ardeur du charbon". C'est cette correspondance qui fait de La chambre double, par exemple, un espace indissociablement intérieur et extérieur, matériel et spirituel:

Une chambre qui ressemble à une rêverie, une chambre véritablement spirituelle, où l'atmosphère stagnante est légèrement teintée de rose et de bleu.

* (BAudelaire, C. Le Poème du haschisch. Op. cit: I, 401.)

(BAUDELAIRE, C. Du vin et du haschisch. Dans CEuvres complètes. Op. cit.: I, 378.)

(FDM L: 49.)

(FDM III: 10.)

'(BAUdelaire, C. Salon de 1846. Op. cit.: II, 482.)

(Ibidem: II, 464.) 
- (BAUDELAIRE, C. Le Spleen de Paris, V. Op. cit.: I, 280.)

(FDM IV: 11)

(FDM LXVII: 48.)

(FDM XXIII: 26.)

- (BAudelaire, C. Mon cœur mis à nu. Op. cit.: I, 702.)
Lâme y prend un bain de paresse, aromatisé par le regret et le désir. - C'est quelque chose de crépusculaire, de bleuâtre et de rosâtre; (...)

Les étoffes parlent une langue muette, comme les fleurs, comme les ciels, comme les soleils couchants. (...)

Une senteur infinitésimale du choix le plus exquis, à laquelle se mêle une très légère humidité, nage dans cette atmosphère.

Cette dernière notation rappelle le rôle, souvent commenté, que joue le parfum dans la création des atmosphères baudelairiennes. Sa subtilité quasi immatérielle en fait une image de l'âme; il a, comme elle, "l'expansion des choses infinies":

Parfois on trouve un vieux flacon qui se souvient D'où jaillit toute vive une âme qui revient.

Mille pensers dormaient, chrysalides funèbres, Frémissant doucement dans les lourdes ténèbres, Qui dégagent leur aile et prennent leur essor, Teintés d'azur, glacés de rose, lamés d'or.

Comme le montre ici la métaphore visuelle qui colore les "pensers" émanés du parfum, l'atmosphère est en fait un ensemble de sensations diverses qui communiquent entre elles par synesthésie. "Les sons et les parfums", en particulier, sont souvent associés dans une même ambiance, comme dans Harmonie du soir. Ils ont en commun une même capacité de remplir l'espace et de pénétrer au plus intime du sujet; Baudelaire fait lui-même de façon explicite ce rapprochement dans $\mathrm{La}$ Chevelure: "Comme d'autres esprits voguent sur la musique,/ Le mien, ô mon amour! nage sur ton parfum". "La musique donne l'idée de l'espace", "creuse le ciel”, et s'avère capable de créer, pour celui qui se laisse porter par elle, une atmosphère propice à l'expression de "toutes les passions":

La musique souvent me prend comme une mer! Vers ma pâle étoile

Sous un plafond de brume ou dans un vaste éther Je mets à la voile; (...)

Je sens vibrer en moi toutes les passions

•(FDM LXIX: 68.)

D'un vaisseau qui souffre $(. . .)^{*}$ 
Cette spatialisation des affects se retrouve dans l'extraordinaire passage de l'essai sur Wagner, où Baudelaire évoque les impressions suscitées en lui par l'audition de l'ouverture de Lohengrin:

Je me peignis involontairement l'état délicieux d'un homme en proie à une grande rêverie dans une solitude absolue, mais une solitude avec un immense horizon et une large lumière diffuse; l'immensitésans autre décor qu'elle-même. (...) Alors je conçus pleinement l'idée d'une âme se mouvant dans un milieu lumineux (...).

Le paysage qui se déploie dans l'esprit de l'auditeur est investi par une Stimmung, qui est à la fois une tonalité musicale, une intensité lumineuse et une résonance affective. Les différentes sensations qui concourent à cette ambiance ne sauraient être dissociées. Rejetant "l'art défini, l'art positif", Baudelaire valorise "l'impression non analysée".*

Il y a chez lui un impressionnisme avant la lettre: il refuse de classer et de hiérarchiser les données sensorielles, pour mieux restituer l'effet global produit par leur réunion. Ainsi le paysage suscité par le parfum de La Chevelure apparaît comme un complexe de sensations multiples:

Tu contiens, mer d'ébène, un éblouissant rêve

De voiles, de rameurs, de flammes et de mâts:

Un port retentissant où mon âme peut boire

À grands flots le parfum, le son et la couleur (...)*

Il s'agit moins d'un paysage composé que d'une ambiance indistinctement visuelle, sonore, olfactive et affective. Cette qualité atmosphérique fait de l'espace affectif un véritable milieu, dans lequel se trouve plongé le sujet lyrique. L'atmosphère l'entoure comme une sphère aux contours imprécis. Le lieu de La Vie antérieure se présente ainsi comme un environnement à la fois naturel et humain où se "mêlent" les sensations les plus diverses:

Les houles, en roulant les images des cieux, Mêlaient d'une façon solennelle et mystique

Les tout-puissants accords de leur riche musique Aux couleurs du couchant reflété par mes yeux.

C'est là que j'ai vécu dans les voluptés calmes, Au milieu de l'azur, des vagues, des splendeurs Et des esclaves nus, tout imprégnés d'odeurs (...)
* (BAUDELAIRE, C. Richard Wagner et Tannhäuser à Paris. Dans CEuvres complètes. Op. cit.: II, 784.)

" (BAudelaire, C. "La chambre double". Le Spleen de Paris, V. Op. cit.: I, 280.)

(FDM XXIII: 26.) 
(FDM III: 10.)

- (BAUDELAIRE, C. Réflexions sur quelques-uns de mes contemporains. Op. cit.: II, 165.)

-(BAUDELAIRE, C. Un mangeur d'opium. Dans $\mathrm{CEu}$ vres complètes. Op. cit.: I, 497-498.)

(BAUdELAIRE, C. Le Peintre de la vie moderne. Dans CEuvres complètes. Op. cit.: II, 690.)

(Idem: II, 253.)

(BAUDELAIRE, C. Un mangeur d'opium. Op. cit.: I, 499.)

(FDM XIX: 23.)

(FDM L: 49)
Ce milieu est souvent présenté ou métaphorisé comme liquide ou aquatique; le sujet lyrique y "baigne" ou y "boit" avec ivresse. "Limmensité profonde" est "une onde" où "l'esprit" se meut et "se pâme" "comme un bon nageur", et où il "boit" la lumière" comme une pure et divine liqueur". "Ces images récurrentes suggèrent que l'atmosphère lyrique garde l'empreinte d'un milieu primitif, qui remonte à une "vie antérieure": si ce n'est prénatale, du moins enfantine. S'il est vrai, comme l'écrit Baudelaire, que "tout poète lyrique" "opère fatalement un retour vers l'Ede perdu", ce "paradis" ne peut être que celui "des amours enfantines". "Dans l'impression atmosphérique que comportent" les plus belles œuvres d'art, le critique perspicace "sent entrer en lui comme une vision de l'enfance de leur auteur". Si "le génie, c'est l'enfance retrouvée à volonté", c'est que, dans les premiers âges de la vie, "les objets enfoncent profondément leurs empreintes dans l'esprit tendre et facile". Et "l'homme qui, dès le commencement, a été longuement baigné dans la molle atmosphère de la femme", * s'avérera plus sensible aux ambiances subtiles et capable de les recréer.

\section{L'espace-corps}

C'est que l'espace affectif du lyrisme est bien sûr à la fois subjectif et intersubjectif. Il est chez Baudelaire fortement marqué par la présence féminine. C'est le mundus muliebris qui donne au poète accès au monde. Comme l'espace et le cœur, le corps amoureux est en expansion. Au cœur-espace correspond un espace-corps agrandi aux dimensions de l'univers, comme celui de $\mathrm{La}$ Géante, contre laquelle le poète rêve de se blottir "comme un hameau paisible au pied d'une montagne". ${ }^{*}$ Cette transformation récurrente de la femme en paysage a souvent pour catalyseur le parfum, capable de franchir les frontières du corps, et de "ravir en extase" le poète. Mais le corps féminin ménage bien d'autres accès à l'espace dont il est le seuil. Dans le regard de la femme en particulier, le poète cherche moins l'expression de sa vie intérieure qu'un reflet du monde qui l'entoure:

On dirait ton regard d'une vapeur couvert; Ton œil mystérieux (est-il bleu, gris, ou vert?) Alternativement tendre, rêveur, cruel, Réfléchit l'indolence et la pâleur du ciel. * 
L'œil ainsi devient ciel, et le visage tend parfois à s'effacer au profit du paysage:

J'aime de vos longs yeux la lumière verdâtre,

Douce beauté, mais tout aujourd'hui m'est amer,

Et rien, ni votre amour, ni le boudoir, ni l'âtre,

Ne me vaut le soleil rayonnant sur la mer.*

À la limite, le femme n'est plus qu'une médiatrice entre le sujet lyrique et l'univers:

Amante ou sœur, soyez la douceur éphémère

D'un glorieux automne ou d'un soleil couchant. (...)

Ah! laissez-moi, mon front posé sur vos genoux,

Goûter, en regrettant l'été blanc et torride,

De l'arrière-saison le rayon jaune et doux!*

"(lbidem)

Il arrive même que les amants disparaissent tous deux, fondus en un pur paysage, qui suffit à exprimer l'atmosphère de leur relation, comme dans les deux premiers vers de Causerie:

Vous êtes un beau ciel d'automne, clair et rose!

Mais la tristesse en moi monte comme la mer (...).

Dans le lyrisme amoureux de Baudelaire, l'évocation du décor et de la circonstance l'emporte souvent sur le récit de la rencontre et sur l'expression des sentiments. Exemplaire est, à cet égard, l'attitude du héros d'un petit poème en prose, Les Projets, qui se borne à imaginer successivement les différents lieux où il pourrait installer sa bien-aimée, et qui sont autant d'atmosphères favorables à une vie commune, probablement destinée à rester à l'état de rêve." Moins ironique, L'Invitation au voyage des Fleurs du Mal repose sur un principe comparable, et confie aux lieux et aux objets du "pays qui (lui) ressemble" le soin de "parl(er) à l'âme" "sa douce langue natale". "Un poème d'amour comme Le Balcon, au titre significatif, nous en dit bien plus sur le décor que sur le corps ou sur le cœur de la femme aimée.

Un bel exemple de ce déplacement des personnages au paysage est fourni par un poème célèbre, tout entier consacré au décor et à l'atmosphère qui entoure une relation dont nous ne connaîtrons ni la nature ni les partenaires:

- (BAUDELAIRE, C. Le Spleen de Paris, XXIV. Op. cit.: I, 314-315.)

"(FDM LIII: 53.) 
Je n'ai pas oublié, voisine de la ville,

Notre blanche maison, petite mais tranquille;

Sa Pomone de plâtre et sa vieille Vénus

Dans un bosquet chétif cachant leurs membres nus,

Et le soleil, le soir, ruisselant et superbe,

Qui, derrière la vitre où se brisait sa gerbe,

Semblait, grand œil ouvert dans le ciel curieux,

Contempler nos dîners longs et silencieux,

Répandant largement ses beaux reflets de cierge

Sur la nappe frugale et les rideaux de serge.

(BAUDELAIRE, C. Réflexions sur quelques-uns de mes contemporains. Op. cit.: II, 165.)
On reconnait ici en général l'évocation de la demeure où Baudelaire et sa mère ont séjourné, à Auteuil, après la mort du général Aupick. Mais cette lecture autobiographique méconnaît le mouvement essentiel du lyrisme, qui tend à dépasser les singularités individuelles pour donner au poème une valeur plus large: "Tout mode lyrique de notre âme nous contraint à considérer les choses non pas sous leur aspect particulier, exceptionnel, mais dans les traits principaux, généraux, universels". " Ici, toute précision de lieu et de date se trouve effacée, et même l'identité et le nombre des habitants de la maison restent indéterminés; ils ne sont désignés que globalement et indirectement par l'adjectif possessif de la $1^{\text {ère }}$ personne du pluriel: ils se résorbent dans l'espace ("notre maison") et dans les repas ("nos dîners") qu'ils partagent.

C'est dans ce qui les entoure qu'il faut chercher les indices de leur intimité. La psychanalyse y voit la projection des fantasmes du poète, qui déplace sur les "membres nus" des statues son désir incestueux, dont l'œil "curieux" du père vient à la fin du poème surveiller, voire censurer, les élans. Cette lecture a sans doute sa pertinence, mais elle aussi trahit la "manière lyrique de sentir". L'espacement du sujet lyrique n'est pas le déplacement à l'extérieur d'un affect refoulé en son for intérieur, mais la dimension même de son ek-sistence, qui se vit et s'ex-prime dans l'espace.

Cet espace est bien sûr aussi un espace poétique, et il faudrait analyser les différents procédés par lesquels Baudelaire parvient à donner à une "petite maison" une dimension cosmique: effacement des repères spatio-temporels, expansion syntaxique de l'unique phrase, qui, par le jeu des incises et des appositions, se développe en membres croissants jusqu'à la fin du poème, amplification ryth- 
mique et sonore, notamment grâce à la diérèse, instrument privilégié de la diction baudelairienne de l'infini. ${ }^{8}$

La forme, dans Les Fleurs du Mal, n'est donc pas si "mesquine" que ne le prétendait Rimbaud. Baudelaire a su faire, des contraintes de la versification régulière le principe d'un approfondissement de l'espace poétique, comme il l'écrit à Fraisse:

Parce que la forme est contraignante, l'idée jaillit plus intense (...) Avez-vous remarqué qu'un morceau de ciel aperçu par un soupirail, ou entre deux cheminées, deux rochers, ou par une arcade, donnait une idée plus profonde de l'infini, qu'un grand panorama vu du haut d'une montagne?*

Commentant cette lettre célèbre, André du Bouchet y voyait l'expression du paradoxe essentielle de la poésie baudelairienne, qui fait de l'obstacle la condition même de son avancée, qui "en repousse" "les limites" "à l'infini". 'Cette recherche de l'infini dans le fini, qui est au cœur de l'esthétique baudelairienne, a aussi une valeur éthique: c'est dans les limites de l'existence et de la réalité quotidiennes que Baudelaire nous invite à découvrir "la profondeur de la vie". Le lyrisme baudelairien ne saurait donc être confondu avec un idéalisme; la transcendance qui l'anime ne vise pas un autre monde: elle est le mouvement par lequel notre monde se révèle autre au sujet qui s'ouvre à lui.

L'espacement du sujet lyrique, que j'ai tenté de mettre au jour dans les Fleurs du Mal, participe d'une tendance profonde de la pensée moderne, qui remet en cause la distinction cartésienne entre la chose pensante et la chose étendue. La phénoménologie existentielle a mis l'accent sur la spatialité de l'être-là et de la conscience comme "être-au-monde" ou comme "champ de présence". Mais elle n'est pas seule à penser le sujet dans l'espace. La topique freudienne repose sur l'hypothèse que "le psychisme lui-même est quelque chose d'étendu", et divers courants de la psychanalyse et de la psychologie contemporaines explorent "l'espace potentiel" où s'opère la transition entre le monde intérieur et le monde extérieur. Aujourd'hui se développe une "écologie de l'esprit" qui étudie ses interactions avec son environnement, que ce soit du côté d'une

\footnotetext{
${ }^{8}$ Voir Michel Deguy, "Linfini et sa diction", dans Choses de la poésie et affaire culturelle (Paris: Hachette, 1986); et Gérald Antoine, "L’expansion sémantique et sonore dans Les Fleurs du Mal”, Études baudelairiennes, VIII (Neuchâtel: La Baconnière, 1976: 9-45).
}

" (BAUDELAIRE, C. "Lettre à Fraisse du 18 février 1860". Dans Correspondance. Bibliothèque de la Pléiade. Paris: Gallimard, 1973: I, 676.)

'(BOUCHET, André du. Baudelaire irrémédiable. Paris: Deyrolle, 1993: 14.) 
-(DELEUZE, Gilles \& GUATTARI, Félix. Qu'est-ce que la philosophie? Paris: Minuit, 1991: 82-108.)

- (SLOTERDIJK, Peter. Bulles, Sphères I. Paris: Pauvert, 2002) "cognition incarnée et située", 9 de la "géophilosophie" esquissée par Deleuze et Guattari* ou de la "sphérologie" élaborée par Sloterdi$\mathrm{jk}$, qui montre que "l'esprit est dans l'espace". En quoi l'expansion lyrique qui se manifeste dans Les Fleurs du Mal ne les rend pas prisonnières de l'héritage romantique; elle a des prolongements dans notre modernité, qui ne se confond plus avec le modernisme et se ressource souvent du côté du romantisme.

${ }_{9}^{9}$ Je fais ici allusion notamment aux travaux de Francisco Varela. 


\section{Michel Collot}

É professor de literatura francesa da Universidade de Paris III, onde dirige a Unidade Mista de Pesquisa "Écritures de la modernité", associada ao CNRS. Publicou inúmeros ensaios sobre a poesia moderna e sobre a paisagem, em especial L'Horizon fabuleux et Paysage et poésie (Corti, 1988 e 2005), La Poésie moderne et la structure d'horizon e La Matière-émotion (PUF, 1989 e 1997); além de coletâneas de poemas, entre as quais Chaosmos (Belin, 1997) e Immuable mobile (La Lettre volée, 2002).

\section{Resumo}

$\mathrm{O}$ artigo discute a herança baudelairiana em suas relações com o romantismo e a modernidade. Para tanto trabalha-se em especial a noção de lirismo, que é dissociada do idealismo com o qual é freqüentemente confundida.

\section{Abstract}

This paper discusses the Baudelairean heritage in its relationship with romanticism and modernity. This is carried out through the notion of lyricism, which is dissociated from the idealism, with which it is frequently confused.

\section{Résumé}

Le lyrisme baudelairien ne saurait donc être confondu avec un idéalisme; la transcendance qui l'anime ne vise pas un autre monde: elle est le mouvement par lequel notre monde se révèle autre au sujet qui s'ouvre à lui.
Palavras-chave: Romantismo; Modernidade; Lirismo; Espacialidade; Charles Baudelaire

Key words: Romanticism; Modernity; Lyrism; Spatiality; Charles Baudelaire

Mots-clés: Romantisme; Modernité; Lyrisme; Spatialité; Charles Baudelaire 